\title{
Schrifttums- und Abkürzungsverzeichnis
}

a. a. 0 .

Adam

Allfeld, LitUG

Allfeld, KunstUG

Amtl. Begr.

Andriztki

Anm.

Art.

Aufl.

Bappert

Bappert-Wagner

Barth

Baum

Bd.

BGB

BGBI

Bem.

Bergner am angegebenen Ort

A d a m, Robert: Zur Lage der Filmwirtschaft in den USA, FuR 1967/51 ff.

A IIf e Id, Philip: Das Urheberrecht an Werken der Literatur und der Tonkunst, Kommentar zu dem Gesetze vom 19. Juni 1901 sowie zu den internationalen Verträgen zum Schutze des Urheberrechts, 2. Aufl., München 1928.

d e r s e I b e : Kommentar zu dem Gesetze vom 9. Januar 1907 betreffend das Urheberrecht an Werken der bildenden Künste und der Photographie, München 1908.

Amtliche Begründung zum Regierungsentwurf eines $\mathrm{Ge}-$ setzes über Urheberrecht und verwandte Schutzrechte (Urheberrechtsgesetz)

Andritzki, Christoph: Die Rechtsstellung des Drehbuchautors, Berlin und Leipzig 1931.

Anmerkung

Artikel

Aufl.

B a p p e rt, Walter: Wege zum Urheberrecht. Frankfurt 1962.

B a p pert-Wagner: Internationales Urheberrecht. Kommentar zur Revidierten Berner Übereinkunft....... von Walter Bappert und Egon Wagner, München und Berlin 1956.

B a rt h Elisabeth: Die Urheberschaft am Film. Diss. jur., Köln 1937.

B a u m, Alfred: Mechanische Musikinstrumente. UFITA Bd. 5 (1932) S. $485 \mathrm{ff}$.

Band

Bürgerliches Gesetzbuch vom 18. 8. 1896 (RGBI. S. $195=$ BGBI III Nr. $400-2$ ).

Bundesgesetzblatt

Bemerkung

B e rg n e r, Heinz: Versuch einer Filmwirtschaftslehre. Filmwirtschaftliche Studien aus dem Industrieseminar der Universität zu Köln.

Bd. 1/I, Berlin 1962

Bd. 1/IV, Berlin 1966. 
Bergström

Berthold/v. Hartlieb

BGH

BGHZ

BJustMin Entwürfe

de Boor

Boytha

Brugger

Bussmann

BTDrucks.

BTProt.

Clair

Dadek,

Filmwirtschaft

Dadek,

Filmmedium
Bergströ m, Svante: Bericht über die Arbeiten der Hauptkommission I der Stockholmer Konferenz von 1967, UFITA-Schriftenreihe, Heft 35 (gebunden, 1969) S. $449 \mathrm{ff}$.

Filmrecht. Ein Handbuch von Friedrich Josef B e r t h o I d und Horst von Hartlie b, München und Berlin 1957.

Bundesgerichtshof

Entscheidungen des Bundesgerichtshofs in Zivilsachen, zitiert nach Bd. und Seite.

Entwürfe des Bundesjustizministeriums zur Urheberrechtsreform, Köln 1959.

d e B o o r, Hans Otto: Urheber- und Verlagsrecht. Ein Beitrag zur Theorie der ausschlieBlichen Rechte. Stuttgart 1917.

B o y th a, György: Urheberrechtliche Konsequenzen der Abhängigkeit der Übersetzung vom Originalwerk. UFITA Bd. 55 (1970) S. $89 \mathrm{ff}$.

B rug g e r, Gustav: Zur Abgrenzung zwischen Fernsehnutzungsrechten und Verfilmungsrechten. UFITA Bd. 33 (1961/l) S. $308 \mathrm{ff}$.

de rse l be: Urheberrechtliche Produktionsfragen des Fernsehens. FuR 1966/89 ff.

derselbe: Der Begriff der Bearbeitung und Verfilmung im neuen Urheberrechtsgesetz. UFITA Bd. 51 (1968) S. $89 \mathrm{ft}$.

B u s s m a n n, Kurt: Anderung und Bearbeitung im Urheberrecht. In: Festschritt für Philipp Möhring zum 65. Geburtstag am 4. 9. 1965, herausgegeben von Prof. Dr. Hefermehl und Prof. Dr. Nipperdey, München und Berlin 1965.

derse l b e : Rechtsprobleme bei Film- und Fernsehen. UFITA Bd. 29 (1959/II), S. $158 \mathrm{ff}$. = UFITA-Schriftenreihe, Heft 15, Aktuelles Filmrecht II (1959) S. 14 ff.

Bundestagsdrucksache, zitiert nach Wahlperiode und laufender Nummer.

Protokolle über die Sitzungen des Deutschen Bundestages, zitiert nach laufender Nummer.

Cla ir, René: Vom Stummfilm zum Tonfilm. Kritische Notizen zur Entwicklungsgeschichte des Film 1920-1950. München und Berlin 1952.

Dadek, Walter: Die Filmwirtschaft. GrundriB einer Theorie der Filmökonomik. Freiburg 1957.

derselbe: Das Filmmedium. Zur Begründung einer Allgemeinen Filmtheorie. München und Basel 1968. 
Degand

Diss. jur., phil.

Dittrich

Eckstein

Elster

engl.

Fagg

Filmhandbuch

Fn.

franz.

Fromm

Fromm-Nordemann

Fromm-Nordemann

1. Aufl.

FuR

G

GBI.

v. Gamm, FilmR

v. Gamm, UG
D e g a n d, Claude: Die Auswirkung des Fernsehens auf die Filmsituation. FuR 1966/ff.

Dissertation der juristischen, philosophischen Fakultät; der zitierte Erscheinungsort ist der Sitz der Universität.

D ittrich, Robert: Die Stockholmer Fassung der Brüsseler Obereinkunft, InterGU Bd. 40 (1968).

Eckstein, Ernst: Deutsches Film- und Kinorecht. Mannheim, Berlin und Leipzig 1924.

E I s te r, Alexander: Urheber- und Erfinderrecht, Warenzeichen- und Wettbewerbsrecht. 2. Aufl., Berlin und Leipzig 1928.

d e rs e l be : Formgebung und Ausdrucksmittel in ihrer Bedeutung für das Recht des Urhebers. UFITA Bd. 2 (1929) S. 595 ff.

englisch

F a g g, John: Urheberschaft und Urheberrecht am Film. Eine kritische Studie. Berlin und Frankfurt/M. 1928.

Filmhandbuch, Neue Ausgabe, Loseblattsammlung, herausgegeben von der Spitzenorganisation der Filmwirtschaft e.V., Berlin und Neuwied.

Fußnote

tranzösisch

F r o m m, Friedrich Karl: Filmrechtsregelung des Urheberrechtsgesetzes. UFITA Bd. 48 (1966/III) S. 21 ff.

From m, Friedrich Karl und Norde m a n Urheberrecht. Kommentar zum Urheberrechtsgesetz und zum Wahrnehmungsgesetz mit den internationalen $A b-$ kommen und den Urheberrechtsgesetzen der DDR, Osterreichs und der Schweiz. 2. durchgesehene und ergänzte Aufl., Stuttgart, Berlin, Köln und Mainz 1970.

di e s e I b e n: Urheberrecht. Kommentar zum Urheberrechtsgesetz und zum Wahrnehmungsgesetz mit den internationalen Abkommen und dem sowjetzonalen Gesetz über das Urheberrecht. 1. Aufl., Stuttgart, Berlin, Köln und Mainz 1966.

Film und Recht, Informationsdienst des Instituts für Filmund Fernsehrecht, München, zitiert nach Jahr und Seite. Gesetz

\section{Gesetzblatt}

von G a m m, Otto Friedrich: Grundfragen des Filmrechts. Berlin und Köln 1957.

d e rs e l be : Urheberrechtsgesetz. Kommentar. München und Berlin 1968. 
Goldbaum

Görgen

Greco

Groll

GRUR

GRUR AIT

Gutbrod

Haake

Haeger

Hagemann

Heinker

Hirsch
G o I d b a u m, Wendez: Urheber- und Urhebervertragsrecht. 2. Aufl., Berlin 1927.

der s e I b e : Tonfilmrecht. Berlin 1929.

G ö rge n, Hermann M.: Die kulturpolitische Bedeutung der Film- und Fernsehforschung. In: Film- und Fernsehfragen. Vorträge der 3. wissenschaftlichen Tagung der Deutschen Gesellschaft für Film- und Fernsehforschung e. V., Emsdetten 1961, S. 1 ff.

G re c o, Paolo: Die Filmwerke. Ihre Struktur und ihre Stellung im Urheberrecht. UFITA-Schriftenreihe, Heft 10 (1958) S. $23 \mathrm{ff}$.

G r o Il, Gunter: Film, die unentdeckte Kunst. München und Berlin 1937.

Gewerblicher Rechtsschutz und Urheberrecht. Zeitschrift der Deutschen Vereinigung für gewerblichen Rechtsschutz und Urheberrecht. Zitiert nach Jahr und Seite.

Gewerblicher Rechtsschutz und Urheberrecht. Auslandsund internationaler Teil.

Gutbrod, Curt Hanno: Von der Filmidee zum Drehbuch. In: Das Manuskript. 2. Aufl., Wilhelmshaven 1960, S. $531 \mathrm{ft}$.

$\mathrm{H}$ a a $\mathrm{ke}$, Wilmont: Literatur und Film. In: Film und Fernsehen im Spiegel der Wissenschaft. Abhandlungen anläBlich des 10-jährigen Bestehens der Deutschen Gesellschaft für Film- und Fernsehforschung. Herausgegeben von Erich Feldmann und Ernst Meier. Gütersloh 1963.

$\mathrm{H}$ a e ger, Siegfried: Gedanken zur Entwicklung eines Gesamturheberrechts für Filmwerke, Fernsehwerke, Hörwerke. UFITA Bd. 22 (1956/II) S. 55 ff.

dersel be : Der Film als Gesamtwerk. UFITA Bd. 25 (1958/I) S. $166 \mathrm{ff}$. = UFITA-Schriftenreihe, Heft 9 (1958).

d e r s e l b e : System und Grundlagen des Filmvertragsrechts. NJW 1959/656 ff.

H a ge ma n n, Walter: Der Film, Wesen und Gestalt. Heidelberg 1952.

He inker, Hans G.: Film und Fernsehen im neuen Urheberrecht. FuR 1966/98 ff.

Hirsch, Ernst E.: Das neue Urheberrechtsgesetz der Türkei. Zugleich ein Beitrag zur Reform des deutschen Urheberrechts. UFITA-Schriftenreihe, Heft 4 (1957).

derselbe: Über die Urheberschaft am Filmwerk. UFITA Bd. 25 (1958/I) S. $5 \mathrm{ff}$.

derselbe: Urheberrecht und verwandte Rechte. UFITA-Schriftenreihe, Heft 11 (1958) S. 8 ff.

de rse I be: Die Werkherrschaft. Ein Beitrag zur Lehre 
Hoffmann

Hubmann

InterGU

Iros

JW

KG

Kohler, Autorrecht

Kohler, Kunstwerk und Autorschutz

Kohler, Urheberrecht

KunstUG

Kummer

v. Laban

Leinveber

Liebeneiner

Lion

Marwitz-Möhring von der Natur der Rechte an Geisteswerken. UFITASchriftenreihe, Heft 26 (1963) S. 19 ff.

ders e I b e : Bearbeitung und Originalwerk. UFITA Bd. $42(1964 /$ II) S. 8 ff.

H offmann, Willy: Gedanken zum Problem des urheberrechtlichen Formschutzes. GRUR 1930/747 ff.

derselbe : Die Berner Übereinkunft zum Schutze von Werken der Literatur und Kunst vom 9. September 1886, revidiert in Berlin am 13. November 1908 und in Rom am 2. Juni 1928. Berlin 1935.

Hub m a n n, Heinrich: Urheber-und Verlagsrecht. 2. Aufl., München und Berlin 1966.

derselbe: Das Filmrecht des deutschen Regierungsentwurts. InterGU Bd. 26 (1962) S. 6 ff.

Schriftenreihe der Internationalen Gesellschaft für Urheberrecht $\theta$. V., Berlin und Frankfurt/M.

I ros, Ernst: Wesen und Dramaturgie des Filmes. Neve, vom Verfasser bearbeitete Aufl., Zürich 1957.

Juristische Wochenschrift, Berlin und Leipzig. Zitiert nach Jahr und Seite.

Kammergericht

Kohler, Josef: Das Autorrecht. Eine zivilistische Abhandlung. Jena 1880.

d e r s e I b e : Das literarische und artistische Kunstwerk und sein Autorschutz. Eine juridisch-ästhetische Studie. Mannheim, Berlin und Leipzig 1892.

derselbe: Das Urheberrecht an Schriftwerken und Verlagsrecht. Stuttgart 1907.

Gesetz betreffend das Urheberrecht an Werken der bildenden Künste und der Photographie vom 9. Januar 1907 (RGBI. S. 7 = BGBI. III $440-3$ ).

K u m m e r. Max: Das urheberrechtlich schützbare Werk. Bern 1968.

von L a b a n, Rudolf: Tanz, Tanzschrift und Urheberrecht. UFITA Bd. 2 (1929) S. $631 \mathrm{ff}$.

Le in ve be r, Gerhard: Urheberschutz des Regisseurs. GRUR 1971/149 f.

L i e b e n e in e $r$, Wolfgang: Spielleiter und Dichter. In: Müller (s. U.) S. 9 ff.

L i o n, E.: Der Schutz gegen die sogenannte Verfilmung von Schriftwerken. GRUR 1927/218 ff.

Das Urheberrecht an Werken der Literatur in Deutschland. Kommentar zum Reichsgesetz vom 19. Juni 1901/ 
Mentha

MinE

Möhring-Nicolini

Möhring-SchulzeUlmer-Zweigert

mschr. vervielf.

Müller

Nehmiz

NJW

Oekonomidis

Peter

RBŌ

Rdz.

RefE
22. Mai 1910 und den internationalen Verträgen Deutschlands. Von Bruno Marwitz und Philipp Möhring, Berlin 1929.

Me $n$th a, Benigne: Film und Fernsehen in der Berner Öbereinkunft. UFITA-Schriftenreihe, Heft 22 (1961) S. 27 ff.

Ministerialentwurf eines Gesetzes über Urhebrerecht und verwandte Schutzrechte (Urheberrechtsgesetz). In: BJust Min Entwürfe (s. o.).

Urheberrechtsgesetz. Erläutert von Philipp Möhring und Käte $\mathrm{Nicolini}$, unter Mitwirkung von Otto $\mathrm{Arzt}$ und Hermann Kroitzs ch, Berlin und Frankfurt/M. 1970.

Quellen des Urheberrechts. Gesetzestext aller Länder und Tabellen über internationale Verträge. Mit systematischen Einführungen. Herausgegeben von Philipp $M$ ö $h$ ring, Erich $S c h u l z e$, Eugen UImer und Konrad $Z$ we i g e rt. Stand: 6. Lieferung 31. 8. 1970.

maschinenschriftlich vervielfältigt

M ü I l e r, Gottfried: Dramaturgie des Theaters, des Hörspiels und des Films. Mit einem Beitrag von Wolfgang Li ebeneiner. 6. erweiterte und verbesserte Aufl., Würzburg 1954.

Nehmiz, Marielouise: Geistige Schöpfung und Tonfilmproblem. Eine Untersuchung auf Grund der deutschen und schweizerischen Gesetzgebungen und des Rechts der Berner Übereinkunft. Diss. jur., Zürich 1935 (mschr. vervielf.).

Neue Juristische Wochenschrift, München und Berlin, zitiert nach Jahr und Seite.

Oekonomidis, Demetrius S.: Zur Regelung des Filmrechts. In: Das Stockholmer Vertragswerk zum Internationalen Urheberrecht, UFITA-Schriftenreihe, Heft 35 (1969), S. $135 \mathrm{ff}$.

Peter, Wilhelm: Zur Abgrenzung zwischen Fernsehsende- und Verfilmungsrechten, UFITA Bd. 34 (1961/II) S. $141 \mathrm{ff}$.

Revidierte Berner Obereinkuntt zum Schutze von Werken der Literatur und Kunst vom 9. September 1886, revidiert in Berlin am 13. November 1908, in Rom am 2. Juni 1928, in Brüssel am 26. Juni 1948 und in Stockholm am 17. Juli 1967.

Randziffer

Referentenentwurf eines Urheberrechtsgesetzes. In: Referentenentwürfe zur Urheberrechtsreform, veröffentlicht durch das Bundesjustizministerium, Bundesanzeiger, Köln 1954. 
RGBI.

RGZ

Riedel

Rehbinder

Roeber

Rspr.

Runge

S.

Samson

Schilling

Schmidt

Schulze

Schulze Rspr.

SchwBG

Siemek

Spaic

s. 0 .

s. $u$.
Reichsgesetzblatt.

Entscheidungen des Reichsgerichts in Zivilsachen, zitiert nach Bd. und Seite.

Rie de I, H.: Die musikalische Bearbeitung. UFITA Bd. 55 (1970) S. $169 \mathrm{ff}$.

Re h b in d e r, Manfred: Das Recht am Arbeitsergebnis. ZSR 1972, S. $1 \mathrm{ff}$.

Ro eber, Georg: Das Filmrecht und seine Reformbedürftigkeit. Berlin 1933.

derselbe: Zum Rechtsbegriff „Filmschaffender“. UFITA Bd. 16 (1942-44) S. $386 \mathrm{ff}$.

derselbe: Der Film im neuen Urheberrecht. FuR 1965/223 ff.

Rechtsprechung

R u n g e, Kurt: Urheber- und Verlagsrecht. Bonn 1948-53.

Seite

S a m s o n, Benvenuto: Das neue Urheberrecht, UFITASchriftenreihe, Heft 32 (1966).

Schilling, Ernst B.: Grundfragen des Tonfilmrechts für Zwangsvollstreckung, Vergleich und Konkurs. Diss. jur., Berlin 1933.

Sch midt, Hannes: Zur Eigengesetzlichkeit epischer und filmischer Gestaltung. In: Film- und Fernsehfragen. Vorträge der 3. wissenschaftlichen Tagung der Deutschen Gesellschaft für Film- und Fernsehforschung e. V., Emsdetten/Westf. 1961.

Schulze, Erich: Kommentar zum deutschen Urheberrecht, Loseblattsammlung, Stand: 3. Lieferung, 28. Februar 1969, Berlin und Frankfurt/M.

de r s e I b e : Stockholmer Konferenz für geistiges Eigentum 1967. InterGU Bd. 39 (1969).

Rechtsprechung zum Urheberrecht. Entscheidungssammlung mit Anmerkungen, herausgegeben von Erich S c h u I $z$ e, München und Berlin. Stand: Oktober 1970.

Schweizerisches Bundesgericht

S i e m e k, Hermann: Der Film als kultursoziologisches Phänomen, seine Physiognomie und seine Wirkung in der Gesellschaft. Diss. phil., Heidelberg 1953. (mschr. vervielt.).

Sp a ic, Vogis lav: Das Urheberrecht und der Film, InterGU Bd. 26 (1962) S. 32 ff.

siehe oben

siehe unten 
XVI

Streicher

Sutermeister

Troller

UFITA

UFITA-Schriftenreihe

Uimer
Streicher, Otto: Grundfragen des Urheberrechts am Filmwerk. Zürich 1950.

Sut ermeister, Peter: Das Urheberrecht am Film. Basel 1955.

T r o I l e r, Alois: Immaterialgüterrecht (Patent-, Marken-, Urheber-, Muster- und Modell-, Wettbewerbsrecht). Bd. I, 2. überarbeitete und erweiterte Auflage, Basel und Stuttgart 1968. Bd. II, Basel und Stuttgart 1962.

von 1928 bis 1945: Archiv für Urheber-, Film- und Theaterrecht, Berlin; ab 1954: Archiv für Urheber-, Film-, Funk- und Theaterrecht, Baden-Baden; ab Bd. 51 (1968): München.

Archiv tür Urheber-, Film-, Funk- und Theaterrecht, Schriftenreihe, Baden-Baden.

U I me r, Eugen: Kinematographie und Urheberrecht. GRUR AIT 1953/182 ff.

ders e I b e : Zum Filmrecht des Entwurfs. GRUR 1954/ $493 \mathrm{ff}$.

derselb e : Grundfragen des Filmrechts. GRUR 1955/ $518 \mathrm{ff}$.

derselbe : Vom deutschen Urheberrecht und seiner Entwicklung. UFITA Bd. 26 (1958/II) S. $257 \mathrm{ff}$.

derselbe : Urheber- und Verlagsrecht. 2. Aufl., Berlin, Göttingen und, Heidelberg 1960.

derselbe: Die Urheberrechtsreform im Lichte der internationalen Rechtsentwicklung. In: Die Urheberrechtsreform. Herausgegeben im Auftrage der Deutschen Studiengesellschaft für Publizistik von Martin Lö ffle r, Schriftenreihe der Deutschen Studiengesellschaft für Publizistik, Bd. 5, München und Berlin 1963.

derselbe: Originalwerk und Bearbeitung im Internationalen Urheberrecht. Festschrift für Walter Bappert: Das Recht am Geistesgut. Studien zum Urheber-, Verlags-, und Presserecht, herausgegeben von Fritz $\mathrm{Ho}$ d e i g e, Freiburg i. Br. 1964, S. 283 ff.

derselbe: Das Urheberrechtsgesetz in den Plänen zur Reform der Berner Obereinkunft. Festschrift für Walter Hallstein: Probleme des Europäischen Rechts. Frankfurt 1966, S. $541 \mathrm{ff}$.

ders e l be : Reimer/Ulmer: Die Reform der materiellrechtlichen Bestimmungen der Berner Obereinkunft, GRUR AIT. 1967/431 ff.

derselbe: Die Filmrechtsregelung in der Stockholmer Fassung der Berner Obereinkunft. FuR 1968/95 ff.

UG

Gesetz über Urheberrecht und verwandte Schutzrechte (Urheberrechtsgesetz) vom 9. September 1965 (BGBI. I S. 1273); letzte Anderung durch Art. 56 des G vom 25. Juni 1969 (BGBI. I S. 645). 
Urt.

Voigtländer-

Elster-Kleine

Werhahn

Wietek

Wipf

Wolff-Raiser

v. Zelewski

ZSR
Urteil

Urheberrecht an den Werken der Literatur und der Ton-

kunst sowie an Werken der bildenden Kunst und der Photographie. Kommentar von Robert Voig t Iän der und Alexander EIste r, 4. neubearb. Aufl. von Hans K I e in e, Berlin 1952.

Werh a h $n$, Jürgen Wolfgang: Der Schöpfer des Filmwerks. Diss. jur., Berlin 1951.

derselbe: Zur Rechtsstellung des Filmregisseurs. UFITA Bd. 19 (1955/I) S. $191 \mathrm{ff}$.

de r s e l b e : Streitfragen der Filmurheberschaft. UFITA Bd. 22 (1956/II) S. $42 \mathrm{ff}$.

Wi e te k, S. M.: Der urheberrechtliche Schutz der Filmund Fernsehwerke (oevres audiovisuelles) in Frankreich und in der Bundesrepublik Deutschland, UFITA Bd. 49 (1967/I) S. $54 \mathrm{ff}$.

W ip f, Rudolf: Die Stellung des Filmregisseurs im Urheberrecht. Zürich 1966.

Sachenrecht. 10. Bearbeitung, von Martin Wolff und Ludwig $R$ a i s e r, Tübingen 1957.

von Zelewski, Armand: Das Urheberrecht auf dem Gebiet der Filmkunst. Emsdetten/Westf. 1935.

Zeitschrift für Schweizerisches Recht, zitiert nach Jahr und Seite. 
